\title{
"Unentschieden" zwischen lokaler Therapie und systemischer Lyse
}

Fragestellung: Ist die endovaskuläre Therapie bei der Behandlung des ischämischen Insults einer systemischen Thrombolyse überlegen?

Hintergrund: Im Moment werden die meisten Patienten mit einem akuten Schlaganfall die keine Kontraindikation haben, systemisch mit Gewebeplasminogenaktivator (rt-PA) im 4,5 Stunden-Zeitfenster behandelt. Seit einiger Zeit gibt es eine Reihe von interventionellen Verfahren, mit denen eine höhere Rekanalisationsrate als mit der systemischen Thrombolyse erreicht wird. Die IMS-III-Studie wollte die endovaskuläre Therapie mit der systemischen Thrombolyse vergleichen.

Patienten und Methodik: In die Studie wurden Patienten eingeschlossen, bei denen innerhalb eines 3-Stunden-Zeitfensters nach Beginn der Symptome eine systemische Thrombolyse mit rt-PA möglich war. Die Randomisierung erfolgte im Verhältnis 2: $1 \mathrm{zu}$ einer interventionellen Therapie oder einer Monotherapie mit rt-PA. Primärer Outcome der Studie war ein Wert auf der modifizierten Rankin-Skala von zwei oder weniger nach 90 Tagen. Bei allen Patienten wurde die systemische Thrombolyse begonnen. Patienten der Interventionsgruppe wurden schnellstmöglich angiografiert und dann nach den lokalen Gegebenheiten entweder mit dem Merci-Retriever, dem Penumbra-System oder gegen Ende der Studie mit dem Solitaire-Device behandelt. Eine weitere Therapieoption war die lokale Gabe von rt-PA über einen Mikrokatheter.

Ergebnisse: Die Studie begann 2006 und wurde im April 2012 vom Sicherheitskomitee wegen einer zu geringen Wahrscheinlichkeit, einen Therapieunterschied zu zeigen, abgebrochen. $\mathrm{Zu}$ diesem Zeitpunkt waren 434 Patienten in der endovaskulären Therapiegruppe und 222 in der systemischen Thrombolysegruppe. Das mittlere Alter der Patienten betrug 68 Jahre, $50 \%$ waren Männer und der mittlere NIHSS-Score betrug 16. Die mittlere Zeit vom Beginn des Schlaganfalls bis zur Initiierung der systemischen Thrombolyse betrug 122 Minuten. Zum Zeitpunkt des Studienabbruchs betrug der Prozentsatz der Patienten mit gutem Outcome 40,8\% in der endovaskulären Therapiegruppe und $38,7 \%$ in der Gruppe die systemisch rt-PA erhielt. Dieser Unterschied war statistisch nicht signifikant. Es gab auch keine Unterschiede für Patienten, die weniger schwer und schwer betrof-

Broderick JP, Palesch YY, Demchuk AM et al; the Interventional Management of Stroke (IMS) III Investigators. Endovascular therapy after intravenous t-PA versus t-PA alone for stroke. N Engl J Med 2013; 368:

893-903 fen waren. Auch für alle sekundären Endpunkte ergab sich kein Unterschied. Die Rekanalisierungsraten in der Interventionsgruppe betrugen $65 \%$ für Verschlüsse der Arteria carotis interna, $81 \%$ für M1-Verschlüsse, $70 \%$ für M2-Verschlüsse und $77 \%$ für multiple M2-Verschlüsse. Der Outcome der Patienten hing von dem Zeitraum bis zur Rekanalisierung und vom Ausmaß der Rekanalisierung ab. Die Mortalität war mit durchschnittlich $11 \%$ innerhalb der ersten sieben Tage in beiden Therapiearmen gleich. Es gab auch keine Unterschiede für intrazerebrale Blutungen oder schwerwiegende Komplikationen. Bei 16\% der Patienten mit interventioneller Therapie kam es zu einer Komplikation wie einem lokalen Hämatom oder einer Perforation des Gefäßes.

Schlussfolgerungen: In der IMS-III-Studie war eine endovaskuläre Therapie einer systemischen Thrombolyse mit rt-PA nicht überlegen.

\section{-Kommentar von Hans-Christoph Diener, Essen}

\section{Ungünstiges Studiendesign}

Die IMS-III-Studie ist ein gutes Beispiel dafür, wie große randomisierte Studien nicht durchgeführt werden sollten. Die Studiendauer war viel zu lang, der Studieneinschluss pro Zentrum viel zu klein und bedingt durch die lange Studiendauer wurde eine Vielzahl von älteren und zuletzt auch neueren Devices zur Thrombektomie benutzt, sodass es keinerlei Möglichkeiten gibt herauszufinden, ob moderne Devices möglicherweise den älteren und insbesondere der lokalen Thrombolyse und Mikrokatheter überlegen sind. Daher müssen die Ergebnisse der jetzt beginnenden Thrombektomie-Studien mit modernsten Stent-Retrievern abgewartet werden, bis endgültig entschieden werden kann, ob es einen therapeutischen Unterschied der Intervention gegenüber der systemischen Thrombolyse gibt.

Anzeige

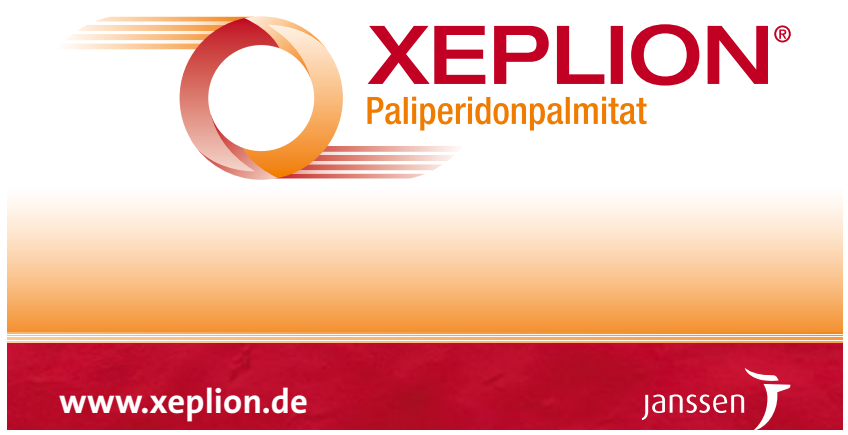

